\title{
Detection of protein aggregation with a Bloch surface wave based sensor
}

\author{
V. Paeder*, V. Musi, L. Hvozdara, S. Herminjard, H.P. Herzig \\ Optics E' Photonics Technology Laboratory, Institute of Microengineering (IMT), Ecole Polytechnique Fédérale de Lausanne (EPFL), A.-L. Breguet 2, 2000 Neuchâtel, Switzerland
}

\section{A R T I C L E I N F O}

\section{Article history:}

Received 30 September 2010

Received in revised form 14 March 2011

Accepted 22 March 2011

Available online 1 April 2011

\section{Keywords:}

Bloch Surface Waves (BSW)

Optical sensor

Protein detection

Protein aggregation

\begin{abstract}
A B S T R A C T
We present the innovative application of a Bloch surface wave based sensor to the detection of protein aggregation. In Hen Egg White Lysozyme (HEWL) solutions, aggregates are discriminated from the monomeric forms in a label-free detection scheme.

(c) 2011 Elsevier B.V. All rights reserved.
\end{abstract}

\section{Introduction}

Multilayer stacks have long been used in a variety of optical applications [1]. Because of their periodic nature, they can be analysed as a one-dimensional case of photonic crystals [2]. One interest of photonic crystals is their ability, when truncated, to support surface states. This ability has been shown to provide guiding at the surface of a terminated multilayer structure [3]. Several authors have since shown experimentally their existence [4] and characterised their properties [5,6]. Moreover, due to the confinement of the wave at the surface, their use in a sensing scheme has been predicted and demonstrated [7-11]. Because of the periodic nature of the underlying structure, these waves are known as Bloch Surface Waves (BSW).

In this study, we present what we believe is, the first demonstration of the application of a BSW-based sensor to the detection of protein aggregation. The process of amyloid fibril formation, due to an incorrect protein recognition pathway, has very important implications in medicine. An increasing number of pathologies are associated unambiguously with the formation of large aggregates of misfolded proteins [12]. Yet are the causes and conditions for which aggregates become pathogenic unclear. Increasing evidences point towards the early stages of protein aggregation. Hence the early dynamic events in the aggregation process are of high interest [13]. The ability to detect and understand these events is of prime importance to tackle many degenerative diseases, which need to be diagnosed prior to the onset of clinical symptoms [14,15].

\footnotetext{
* Corresponding author. Tel.: +41 3271832 81; fax: +41 327183201.

E-mail address: vincent.paeder@epfl.ch (V. Paeder).
}

In this context, BSW sensors, as well as surface plasmon resonance (SPR) sensors [16], have unique properties, such as real time response, versatility, low cost and extremely high surface sensitivity. The ability of the former to detect various protein interactions has been already demonstrated [17-19]. In contrast to SPR sensors, BSW sensors are metal free. They consist exclusively of dielectric materials, providing additional design freedom, long range guiding properties, improved wear resistance and they are inherantly inert with respect to sensitive biological materials. For certain applications, the absence of damping in dielectrics with resulting narrower resonances and the possibility to engineer the dielectric stack to tailor the surface waves dispersion can provide a relevant improvement.

This article is organised as follows. A brief theoretical introduction to BSW is presented in Section 2. The fabrication is detailed in Section 3. The sample preparation and measurement results are presented in Section 4. Finally, a discussion of the results, possible improvement paths and interest over SPR sensors are layed out in Section 5.

\section{Theoretical background}

BSW can be excited on an appropriately truncated periodic structure exhibiting a forbidden band at the working light frequency. In a semi-infinite configuration, a sufficient condition under which such waves appear is simple: the layer at the surface must be tuned to support a propagating mode in the forbidden band of the underlying structure. Any kind of finite periodic structure can thus potentially support a BSW. For the sake of simplicity, we limit our study to one-dimensional dielectric stacks. 


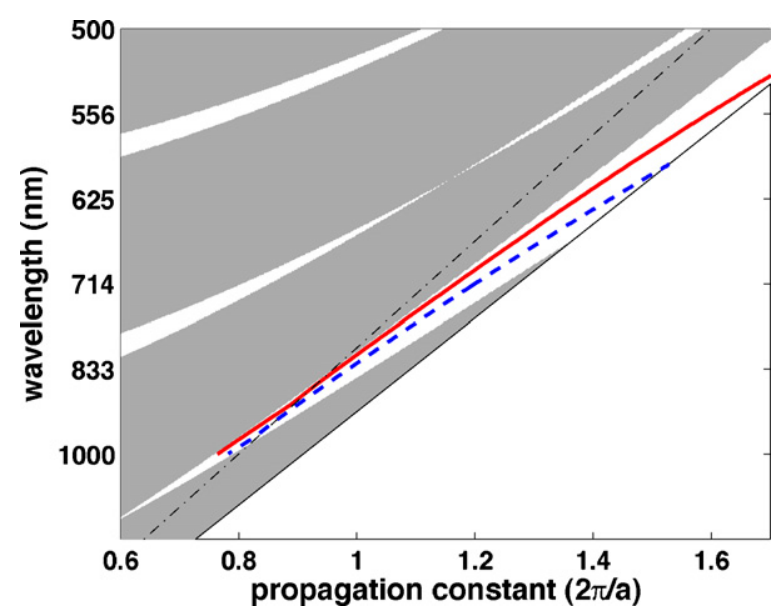

Fig. 1. Band diagram of the structure for TM polarisation, with $a=t_{L}+t_{H}$. The white areas are forbidden bands. Solid red line: defect mode for $t_{g}=290 \mathrm{~nm}$; dashed blue line: $t_{g}=350 \mathrm{~nm}$. The black lines are the light lines in the incident medium (solid line) and in water (dash-dotted line). (For interpretation of the references to color in this figure legend, the reader is referred to the web version of the article.)

The case under study is a multilayer structure made of 9 alternating layers of silicon nitride $\left(\mathrm{Si}_{3} \mathrm{~N}_{4}\right.$, refractive index $n_{H}=1.95$ at $\lambda=632.8 \mathrm{~nm})$ and silicon dioxide $\left(\mathrm{SiO}_{2}, n_{L}=1.47\right)$. With correct parameters, the first 8 layers form a Bragg reflector (BR) which confines a guided mode to the last layer. To operate at $\lambda=632.8 \mathrm{~nm}$, we choose thicknesses $t_{H}=119 \mathrm{~nm}\left(\mathrm{Si}_{3} \mathrm{~N}_{4}\right)$ and $t_{\mathrm{L}}=474 \mathrm{~nm}\left(\mathrm{SiO}_{2}\right)$.

The band diagram of the structure is shown in Fig. 1 for TM polarisation (magnetic field vector parallel to the layers). The position of the surface mode within the bandgap is chosen by tuning the thickness $t_{g}$ of the last layer. We use the method proposed in [20] to match $t_{g}$ to specific resonance conditions.

To be effectively confined at the surface, the mode should lie below the light line of the adjacent medium. This corresponds to a total internal reflection (TIR) condition, as required for SPR sensing.

For sensing, the electric field enhancement at the surface and the penetration depth in the probed medium can be adjusted depending on the distance of the defect mode to the band edge and the light line, respectively. The closer to the light line, the less confined the field is, allowing to choose between surface and volume sensing. The position within the bandgap influences both coupling efficiency and field enhancement. By reciprocity, the coupling efficiency is equivalent to the leakage losses through the BR, and is linked to the imaginary part of the mode propagation constant. Both field enhancement and coupling efficiency are linked to the quality factor ( $Q$ factor) of the resonant mode, which governs the spectral width of the resonance.

\subsection{Sensing in Kretschmann configuration}

As shown in Fig. 2, this configuration consists in illuminating the multilayer in TIR, from a high index medium, under the coupling conditions to the BSW. As for SPR, the coupling is observed as an intensity dip in the reflection spectrum. Changes in the index of refraction near the surface produce a shift of the dip position in the spectrum. Numerically, we compute the reflection spectrum using a Transfer Matrix Method (TMM) algorithm adapted from [21].

In a typical SPR experiment, the sharpest possible dip is sought, as it brings higher accuracy in discriminating the dip shift. This is the case as the field is located at the interface between the metal and the probed medium. Therefore, for a given dip strength, an increased $Q$ factor improves the sensitivity. The attainable $Q$ factor and the dip strength are limited by the absorption in the metal and the coupling efficiency.

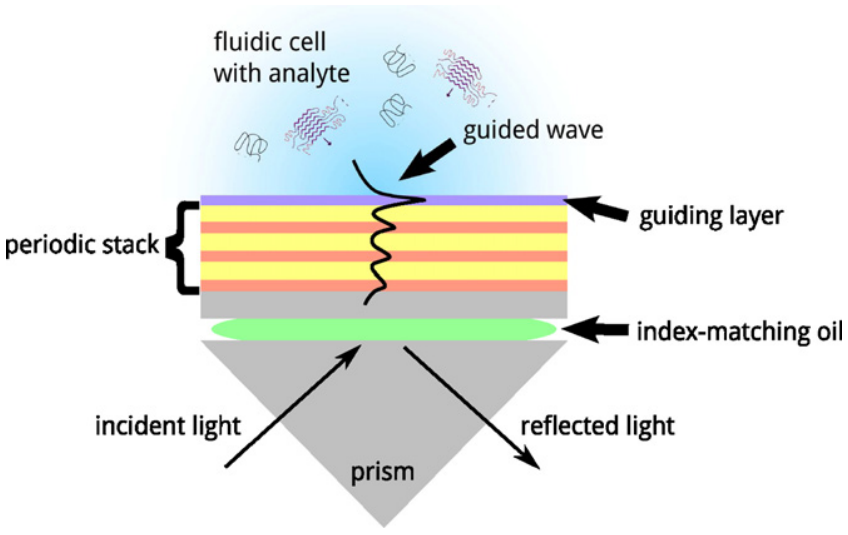

Fig. 2. Schematic representation of the BSW-based sensing scheme in Kretschmann configuration.

For BSW sensing, the intrinsic material absorption is negligible. The presence of a dip in the reflection spectrum is caused by scattering losses such as surface roughness and material imperfections, which scatter the surface wave in all directions. These losses are small and not obvious to measure accurately. They however have an important influence on the dip strength and the coupling efficiency. In the simulation, the scattering losses are approximated as an artificial material absorption. As shown in Fig. 3, the strongest dip is obtained if the scattering losses are comparable to the leakage losses. Here, the BR design angle specifies the angle used to compute the quarter-wavelength thicknesses for the high and low

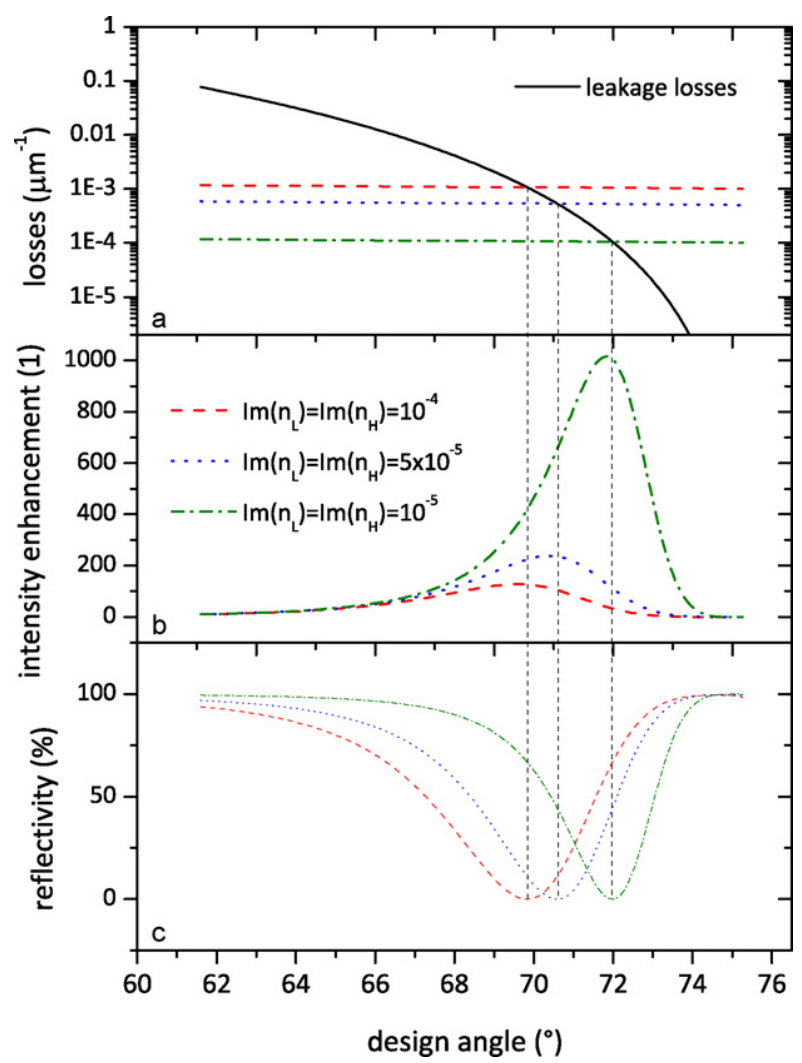

Fig. 3. Influence of the scattering losses on BSW excitation for three values of artificial absorption, as a function of the multilayer design angle: (a) comparison of scattering losses to leakage losses, (b) related intensity enhancement at the surface, and (c) reflectivity. The best dip strength is obtained when the coupling losses and the scattering losses are equal. The black dashed vertical lines highlight the relationship between the quantities. 


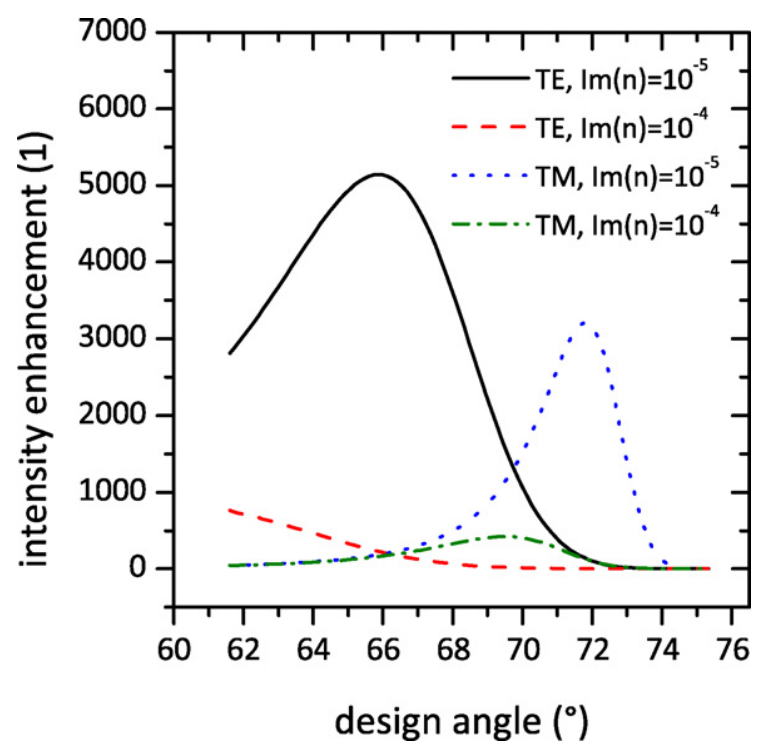

Fig.4. Field enhancement as a function of the design angle, for two values of artificial material absorption and both polarisations.

index layers. The leakage losses are computed with the method presented in [20] taking into account the finite nature of the experimental structure. This condition closely correspond to the case of highest field enhancement at the surface. In the design of the structure used in our experiment, a coarse estimate for the imaginary part of the refractive indices, $\operatorname{Im}\left(n_{L}\right)=\operatorname{Im}\left(n_{H}\right)=10^{-4}$, has been used, based on ellipsometric measurements. In this simplified scheme, absorption and scattering losses are included in a single term.

Moreover, the geometry of the structure has important consequences. The $\mathrm{Q}$ factor of the BSW resonance increases with the number of layers in the stack, while the coupling efficiency decreases. However, as the surface wave behaves as a guided mode in the top layer, the maximum field intensity is not automatically located at the surface. The presence of the underlying BR permits the top layer to be much thinner than the cut-off thickness of a conventional waveguide, enhancing the evanescent field in the probed medium. Varying its thickness influences the distribution of the electric field of the guided mode among the top layer and the probed medium. A strong polarisation dependence can be observed.

Fig. 4 shows the field enhancement for TE and TM polarisations as a function of the design angle, in the presence of a small artificial absorption, for a 9 layer structure as described above. In both cases, the intensity enhancement grows until the BR becomes thick enough to let the scattering losses dominate. The difference between polarisations resides in the continuity conditions affecting the electric field. In TE polarisation, the electric field is continuous across the interfaces, and is preferentially confined to the high index medium. In TM polarisation, the electric field is discontinuous across the interfaces. Due to the index contrast, the field amplitude is higher on the low index side. Because of the larger thickness required for low index regions, the field experiences less interaction with interface roughness. The optimal design angle is thus larger for TM polarisation, providing a shorter evanescent decay near the surface. The optimal design angle is thus larger for TM polarisation, providing a shorter evanescent decay near the surface.

For our experiment, the TIR condition between the prism and water imposes a large working angle. In addition to the aforementioned property, the TM polarisation benefits of a reduced reflection at the air-prism interface, up to Brewster's angle. We therefore choose to work in this configuration.

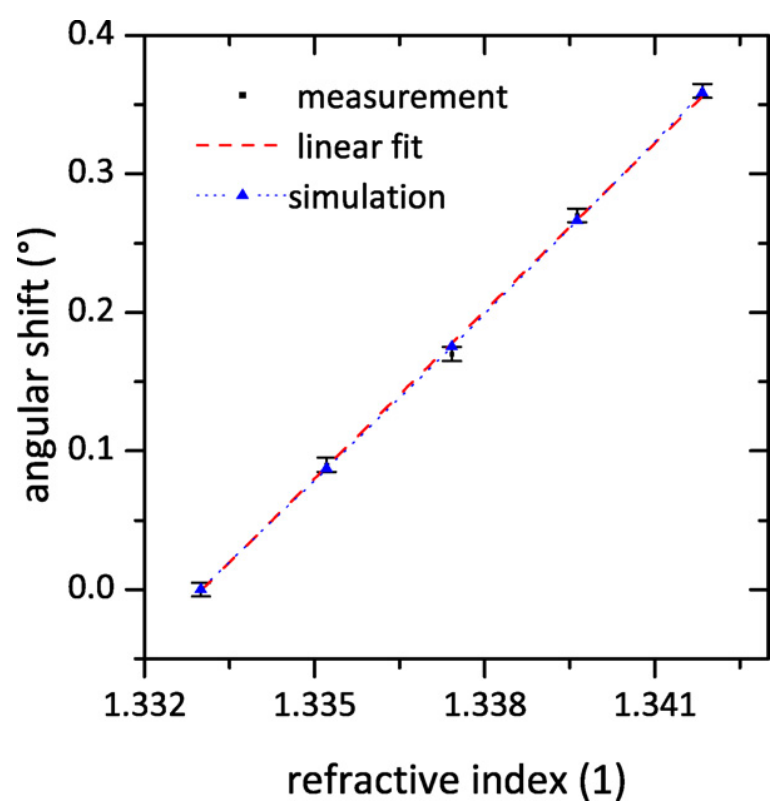

Fig. 5. Measured calibration curve with several concentrations of isopropanol in water. The sensor response is linear with a slope $S_{\theta}=40.35 \pm 0.69^{\circ} / \mathrm{RIU}$.

\section{Sensor fabrication and characterisation}

The multilayer is deposited with an Oxford Plasmalab 80+ PECVD machine. The layer parameters are chosen from simulations to give a resonance in TM polarisation. As mentioned previously, resonances in TM polarisation give more sensitivity at a large incident angle. Moreover, scattering losses introduced by surface roughness impose an optimal working angle. In practice, an upper limit is thus imposed by the deposition method and parameters. A resonance angle $\theta=70.40^{\circ}$ leading to technologically suitable thicknesses is thus taken. The associated thicknesses are $t_{H}=119 \mathrm{~nm}, t_{L}=474 \mathrm{~nm}$ and $t_{g}=291 \mathrm{~nm}$. Measurements in crosssection with a scanning electron microscope gave the values $t_{H}=120 \pm 8 \mathrm{~nm}, t_{L}=486 \pm 4 \mathrm{~nm}$ and $t_{g}=290 \mathrm{~nm}$. The resulting resonance angle is $\theta=71.03^{\circ}$, in close agreement with the theoretical prediction.

The sample is contacted with an index matching oil on a right angle glass prism $(n=1.5149)$. The sensitivity is evaluated with several concentrations $(0-20 \%)$ of isopropanol $(n=1.3772)$ in water $(n=1.3330)$. A linear dependence of the refractive index on concentration is observed. The calibration curve is shown in Fig. 5. It agrees well with the simulation results, corrected for the refraction at the air-prism interface. The angular sensitivity is $S_{\theta}=\Delta \theta \mid \Delta n=40.35 \pm 0.69^{\circ}$ per Refractive Index Unit (RIU). The resolution of the system is $\delta n=1.24 \times 10^{-4}$ RIU. This is two orders of magnitude lower than the best SPR sensors [16], but is inherent to the design choices rather than the technique, that has been shown to reach much larger sensitivities [8]. The lower observed resolution is due to the angular resolution of the motors used in the angular scanning scheme.

\section{Measurement and results}

The experiment is conducted on wild type Hen Egg White Lysozymes (HEWL), the amyloid fibrils of which are a largely described model system [22]. HEWL samples are dissolved in concentrations from $35 \mu \mathrm{M}$ to $1.4 \mathrm{mM}(0.5 \mathrm{mg} / \mathrm{ml}$ to $20 \mathrm{mg} / \mathrm{ml})$ in a $10^{-2} \mathrm{M} \mathrm{HCl}$ solution ( $\mathrm{pH} 2$ ). In order to generate lysozyme fibers, the mixtures are incubated for 1 week at $65^{\circ} \mathrm{C}$ [23]. 


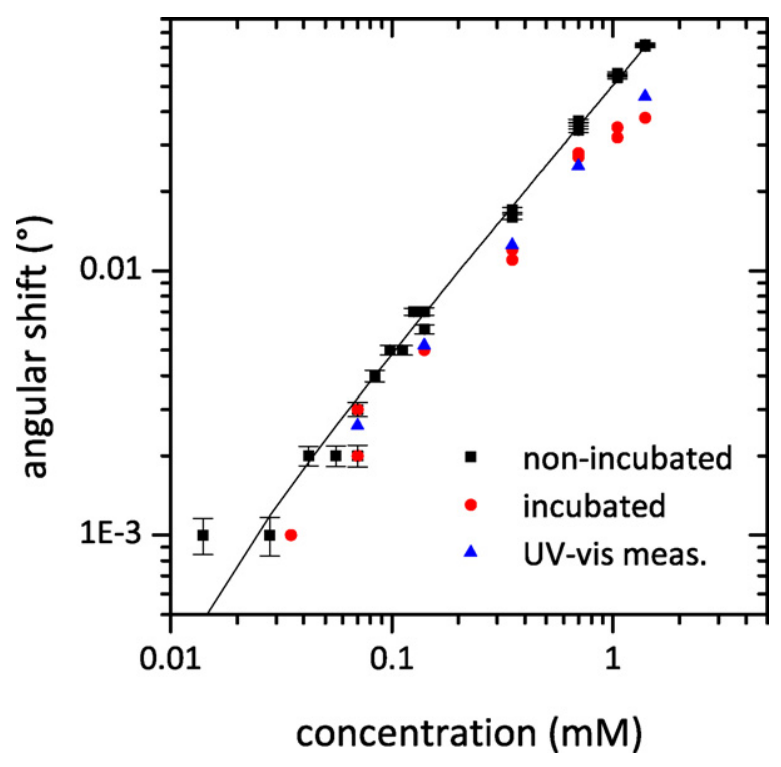

Fig. 6. Measurement of the angular shift produced by several concentrations of non-aggregated (black squares) and aggregated (red circles) proteins. The black line shows a least square fitting for the non-aggregated samples. The blue triangles are the expected shift from the concentration measured with the UV-vis spectrometer and mapped with the linear relationship specified in the text. (For interpretation of the references to color in this figure legend, the reader is referred to the web version of the article.)

The protein concentrations are determined by absorption measurements at $280 \mathrm{~nm}$ before and after incubating the samples. The presence of protein fibrils is confirmed through electron microscopy.

The protein samples are then applied to the optical sensor where a comparison between non-incubated and incubated solutions is conducted. The sensor surface is not functionalised, which implies that the spatial distribution of the aggregates is not forced by strongly and selectively binding the proteins to the surface.

As emphasized in Fig. 6, the non-incubated solutions show a linear relationship $\Delta \theta=S_{C} C$ with the protein concentration $C$, where $S_{C}=5.07 \times 10^{-2} \pm 7.42 \times 10^{-4}$ is computed with a least square fitting. The incubated solutions show a reduced angular shift that increases with concentration. The sensor is able to detect the concentration of the protein in its monomeric form. During aggregation, this concentration is decreasing as the ratio of monomeric to aggregated form increases. The kinetics of amyloid fibril formation is a function of the protein concentration. Low protein concentration slow down the aggregation kinetics substantially, which explains the proportionally reduced shift [24].

At present, the sensor is capable of detecting aggregates at a minimum concentration of $30 \mu \mathrm{M}$ in $110 \mu \mathrm{M}$ of monomeric protein. The detection scheme is completely label-free. The biomolecules are unlabelled (chemically unmodified) and are detected in their natural form. Moreover, the sensor has not been functionalized. The advantage of the label-free concept is the elimination of detrimental effects from labels that may intefere with fundamental interactions and the absence of a time-consuming pre-treatment. The sensor is sensitive to concentration variations that the aggregation process produces rather than to the specific aggregates. Measurements of protein concentration with a UV-vis spectrometer are performed as a control procedure. The determined concentration is then converted to an expected shift with the linear relationship computed from the non-incubated cases. The resulting values for incubated samples, presented as blue triangles in Fig. 6, show a good correlation.

\section{Discussion and conclusion}

We have shown for the first time the possibility to distinguish the protein aggregates, with a BSW-based sensor, in a label-free scheme. The lowest HEWL concentration in which aggregates are discriminated is $140 \mu \mathrm{M}$. The sensor is able to detect proteins near to physiological concentrations (3.5 $\mu \mathrm{M}$ or lower) [25]. However, the discrimination of fibril formation at the very early stages of aggregation is only achieved at higher concentrations.

The sensitivity is mainly limited by the choice of polarisation (TM). As it has been shown, the working conditions in TM provide a larger surface sensitivity, through a larger working angle. However, the field enhancement is lower than in TE. A careful design including optimisations of material parameters (refractive indices, scattering losses), structure and prism material will bring substantial improvements in future works. A possible surface functionalisation providing selectivity towards aggregates [26] is also compatible.

Other features specific to BSW, such as long-range guiding of surface waves [27], are of particular interest. The ability to use alternative materials as a guiding layer, such as teflon, or other biologically inert substances, is an important advantage with respect to SPR based sensors. Owing to their additional freedom in materials and design parameters, their ease of use and their cost efficiency, BSW present a valuable and promising tool for biological and medical sensing applications.

\section{References}

[1] C. Elachi, Waves in active and passive periodic structures-a review, IEEE Proceedings 64 (1976) 1666-1698.

[2] J.D. Joannopoulos, S.G. Johnson, J.N. Winn, R.D. Meade, Photonic Crystals: Molding the Flow of Light, 2nd ed., Princeton University Press, 2008.

[3] P. Yeh, A. Yariv, A. Cho, Optical surface waves in periodic layered media, Applied Physics Letters 32 (1978) 104.

[4] W. Robertson, M. May, Surface electromagnetic wave excitation on onedimensional photonic band-gap arrays, Applied Physics Letters 74 (1999) 1800.

[5] E. Descrovi, T.Sfez, L. Dominici, W. Nakagawa, F. Michelotti, F. Giorgis, H. Herzig, Near-field imaging of Bloch surface waves on silicon nitride one-dimensional photonic crystals, Optics Express 16 (8) (2008) 5453-5464.

[6] T. Sfez, E. Descrovi, L. Yu, M. Quaglio, L. Dominici, W. Nakagawa, F. Michelotti, F. Giorgis, H. Herzig, Two-dimensional optics on silicon nitride multilayer: refraction of Bloch surface waves, Applied Physics Letters 96 (2010) 151101.

[7] M. Liscidini, J. Sipe, Analysis of Bloch-surface-wave assisted diffraction-based biosensors, Journal of the Optical Society of America B 26 (2) (2009) 279-289.

[8] F. Giorgis, E. Descrovi, C. Summonte, L. Dominici, F. Michelotti, Experimental determination of the sensitivity of Bloch surface waves based sensors, Optics Express 18 (8) (2010) 8087-8093.

[9] H. Qiao, B. Guan, J.J. Gooding, P.J. Reece, Protease detection using a porous silicon based bloch surface wave optical biosensor, Optics Express 18 (14) (2010) 15174-15182.

[10] V. Konopsky, E. Alieva, Photonic crystal surface waves for optical biosensors, Analytical Chemistry 79 (12) (2007) 4729-4735.

[11] F. Michelotti, B. Sciacca, L. Dominici, M. Quaglio, E. Descrovi, F. Giorgis, F. Geobaldo, Fast optical vapour sensing by Bloch surface waves on porous silicon membranes, Physical Chemistry Chemical Physics (PCCP) 12 (2) (2010) 502.

[12] F. Chiti, C.M. Dobson, Protein misfolding, functional amyloid, and human disease, Annual Review of Biochemistry 75 (1) (2006) 333-366.

[13] M. Bucciantini, E. Giannoni, F. Chiti, F. Baroni, L. Formigli, J. Zurdo, N. Taddei, G. Ramponi, C. Dobson, M. Stefani, Inherent toxicity of aggregates implies a common mechanism for protein misfolding diseases, Nature 416 (6880)(2002) 507-511.

[14] P. Lansbury, H. Lashuel, A century-old debate on protein aggregation and neurodegeneration enters the clinic, Nature 443 (7113) (2006) 774-779.

[15] S. Baglioni, F. Casamenti, M. Bucciantini, L. Luheshi, N. Taddei, F. Chiti, C. Dobson, M. Stefani, Prefibrillar amyloid aggregates could be generic toxins in higher organisms, Journal of Neuroscience 26 (31) (2006) 8160.

[16] M. Piliarik, J. Homola, Surface plasmon resonance (spr) sensors: approaching their limits? Optics Express 17 (19) (2009) 16505-16517.

[17] J. Homola, Surface plasmon resonance sensors for detection of chemical and biological species, Chemical Review 108 (2) (2008) 462-493.

[18] S. Choi, Y. Yang, J. Chae, Surface plasmon resonance protein sensor using Vroman effect, Biosensors and Bioelectronics 24 (4) (2008) 893-899.

[19] M. Aguilar, D. Small, Surface plasmon resonance for the analysis of $\beta$-amyloid interactions and fibril formation in Alzheimer's disease research, Neurotoxicity Research 7 (1) (2005) 17-27. 
[20] Y.-F. Li, J.W.Y. Lit, General formulas for the guiding properties of a multilayer slab waveguide, Journal of the Optical Society of America A 4 (4) (1987) 671-677.

[21] L. Li, Reformulation of the Fourier modal method for surface-relief gratings made with anisotropic materials, Journal of Modern Optics 45 (7) (1998) 1313-1334.

[22] G. Merlini, V. Bellotti, Lysozyme: a paradigmatic molecule for the investigation of protein structure, function and misfolding, Clinica Chimica Acta 357 (2) (2005) 168-172.

[23] L Morozova-Roche, J. Zurdo, A. Spencer, W. Noppe, V. Receveur, D. Archer, M. Joniau, C. Dobson, Amyloid fibril formation and seeding by wild-type human lysozyme and its disease-related mutational variants, Journal of Structural Biology $130(2-3)(2000) 339-351$.

[24] Y. Georgalis, P. Umbach, J. Raptis, W. Saenger, Lysozyme aggregation studied by light scattering. II. Variations of protein concentration, Acta Crystallographica Section D: Biological Crystallography 53 (6) (1997) 703-712.

[25] A.K.H. Cheng, B. Ge, H.-Z. Yu, Aptamer-based biosensors for label-free voltammetric detection of lysozyme, Analytical Chemistry 79 (14) (2007) 51585164.

[26] M.-J. Bañuls, V. González-Pedro, C.A. Barrios, R. Puchades, Á. Maquieira, Selective chemical modification of silicon nitride/silicon oxide nanostructures to develop label-free biosensors, Biosensors and Bioelectronics 25 (6) (2010) $1460-1466$.

[27] E. Descrovi, T. Sfez, M. Quaglio, D. Brunazzo, L. Dominici, F. Michelotti, H. Herzig, O. Martin, F. Giorgis, Guided Bloch surface waves on ultrathin polymeric ridges, Nano Letters 10 (6) (2010) 2087-2091.

\section{Biographies}

Vincent Paeder received the MS degree from the University of Neuchatel, Switzerland, in 2006, and is now working toward the PhD in the Optics and Photonics Technology Laboratory of the Ecole Polytechnique Federale de Lausanne (EPFL). His current research interests include the simulation of nanostructured optical devices and the application of multilayered structures to actuation, wave guiding and biosensing.

Valeria Musi has a long-term expertise in the field of protein chemistry and structural biology. She acquired substantial experience on chemical-analytical techniques for protein analysis and optical spectroscopies. During the undergraduate thesis in chemistry (Padua, Italy), she worked on the study of protein folding and stability using biochemical and biophysical techniques. At the National Institute for Medical Research in London (division of Molecular Structure), during her PhD and post-doctoral experience, structural biology and biophysics became her main subjects while studying the structural basis of molecular recognition by NMR and
Optical spectroscopies. Since the end of 2008 she is at the IMT in the Optics \& Photonics Technology Laboratory as project leader on the development of biosensors for biophotonics studies, investigating optical detection methods that can be applied to biological systems with a particular interest in protein interactions and aggregation.

Lubos Hvozdarara (1972) has Graduated in Electrical engineering from Slovak University of Technology Bratislava (SK) in 1996 MBE. He received his PhD degree from Vienna University of Technology in Vienna (A) in 2000. His PhD program was dedicated to GaAs/AlGaAa based Quantum Cascade Lasers for Spectroscopy. In 2001 he joined the School of Chemistry and Biochemistry of Georgia Institute of Technology in Atlanta (GA) as a post-doctoral fellow. He developed applied laser spectroscopic systems for marine environment. In 2002 he assumed a post of a Technology manager at Alpes Lasers in Neuchatel $(\mathrm{CH})$ - world leading producer of Quantum Cascade Lasers. In 2009 he joined the group of applied optics as a Project leader, dedicating his research to various mid-infrared lasers applications. Currently he works laser application for spectroscopy of human bodily fluids.

Sylvain Herminjard received the master of micro-engineering from the Ecole Polytechnique Federale de Lausanne (EPFL) in 2005 and the Ph.D. degree in optics from the same institution in 2010. During his academic activities, his research interests were mainly surface plasmon resonance techniques applied to sensing and monitoring applications in the mid-infrared range of the electromagnetic spectrum. He also worked on the design of high-sensitivity sensors based on surface waves technique applied to biological applications. He is now working as a R\&D engineer in a company that develops highly specialized quantum cascade laser sources for sensing applications.

Hans Peter Herzig received the Diploma degree in physics from the Swiss Federal Institute of Technology, Zurich, Switzerland, in 1978, and the Ph.D. degree in optics from the University of Neuchâtel, in 1987. From 1978 to 1982, he was a Scientist with the Department of Optics Development of Kern, Aarau, Switzerland, working in lens design and optical testing. In 1983, he was a Graduate Research Assistant with the Applied Optics Group, Institute of Microtechnology, University of Neuchâtel, Switzerland, working in the field of holographic optical elements. From 1989 to 2001, he was the Head of the Micro-Optics Research Group. From 2002 to 2008, he was a Full Professor and the Head of the Applied Optics Laboratory, University of Neuchâtel. He joined the faculty with the École Polytechnique Fédérale de Lausanne (EPFL), Switzerland, in 2009. Currently, he is a Professor with the EPFL. His research interests include refractive and diffractive micro-optics, nano-scale optics, and optical MEMS. Dr. Herzig is a member of the OSA, IEEE Photonics Society, EOS, and SSOM 2009/2010 he was President of the European Optical Society (EOS). He served as the Conference Chairman for international conferences of EOS, IEE, IEEE/LEOS, OSA and SPIE, and as a Guest Editor of four special issues of IEEE and OSA journals. He is the Editor of a well-known book on micro-optics (published in English and Chinese), author of 12 book chapters, over 120 journal papers and 250 conference proceedings. 